\title{
Evaluación del Proceso de Enseñanza-Aprendizaje sobre Fuentes Renovables de Energía desde los Paradigmas Cognitivo y Ecológico-Contextual
}

\author{
Andrea P. Rossi ${ }^{1}$, Horacio R. Di Prátula ${ }^{1}$ y Alejandro J. Vitale $e^{1,2,3}$ \\ (1) Universidad Tecnológica Nacional, Facultad Regional Bahía Blanca, Departamento de \\ Ingeniería Eléctrica (UTN FRBB), GESE (Grupo de Estudios Sobre Energía), 11 de Abril 461, \\ B8000LMI Bahía Blanca, Buenos Aires, Argentina (email: aprossi@frbb.utn.edu.ar, \\ hrdiprat@bblanca.com.ar) \\ (2) Instituto Argentino de Oceanografía, Camino La Carrindanga Km. 7,5 CC 804, B8000FWB, \\ Bahía Blanca, Buenos Aires, Argentina. \\ (3) Universidad Nacional del Sur, Departamento de Ingeniería Eléctrica y de Computadoras, Avda. \\ Alem 1253, 8000 Bahía Blanca, Buenos Aires, Argentina (email: avitale@criba.edu.ar)
}

\section{Resumen}

En el presente trabajo se presenta una nueva metodología para la evaluación de la eficacia del proceso de enseñanza-aprendizaje en carreras de Ingeniería. Esta ha sido diseñada considerando los criterios cognitivo constructivista y ecológico-contextual no utilizados comúnmente en carreras de ingeniería ni en asignaturas de contenido técnico. La metodología utiliza no sólo los resultados de las evaluaciones de los alumnos, sino también una herramienta de evaluación de cátedra consistente en una planilla a ser completada por los estudiantes con sus opiniones, una vez finalizada la etapa de evaluación de la asignatura. La nueva herramienta se aplicó a dos asignaturas de la carrera Ingeniería Eléctrica y arrojó resultados notablemente favorables, de acuerdo a los indicadores de medición elegidos. Se prevé continuar con la nueva modalidad en ambas asignaturas, a fin de realizar un seguimiento con diferentes grupos de alumnos, obtener mayor cantidad de datos y asegurar la confiabilidad de los resultados.

\section{Evaluation of the Teaching-Learning Process on Renewable Energy Sources from Cognitive and Ecological-Contextual Paradigms}

\begin{abstract}
The present paper presents a new methodology for assessing the effectiveness of the teachinglearning process in engineering careers. This has been designed by considering the cognitive and ecological approaches not commonly used in engineering careers nor in technical courses. The methodology considers not only the results of the grades obtained by the students but also an evaluation tool consisting of a questionnaire form to be completed by students once the stage of evaluation of the course is done. The new tool was applied to two courses of Electrical Engineering and gave remarkably favorable results, according to the indicators used. It is expected to continue with the new approach in both courses in order to track different groups of students and to obtain more data to ensure reliability of the results.
\end{abstract}

Keywords: assessment, teaching and learning, renewable energy, cognitive paradigm, ecologicalcontext paradigm 


\section{INTRODUCCIÓN}

La eficacia del proceso de enseñanza-aprendizaje en carreras de Ingeniería es usualmente medido a través de la sola evaluación del aprendizaje del alumno (Bloom et. al, 1982). Los mecanismos desarrollados durante la intervención didáctica quedan así fuera de la evaluación del proceso educativo, dificultando la identificación de los errores cometidos en la aplicación de la herramienta elegida para la enseñanza (Blanco Gutiérrez, 2004).

Aún cuando la herramienta contemple una modalidad de evaluación del alumno que facilite el seguimiento cercano del proceso enseñanza-aprendizaje, si dicho seguimiento sólo está basado en el análisis de las calificaciones de los alumnos y/o en la observación del logro de objetivos fijados por la cátedra (y ambos realizados únicamente por parte del docente), no se estará teniendo real conocimiento de si la metodología con que los contenidos fueron impartidos resulta ser la más adecuada para la temática desde la percepción y experiencia del estudiante.

Es así que se hace necesario un método de análisis que contemple las opiniones que puedan aportar los alumnos mediante evaluaciones de cátedra (Gonzáles, 1996). Con esta premisa, en el primer cuatrimestre del año 2007 se implementó en la asignatura "Fuentes No Convencionales de la Energía" (hoy denominada "Fuentes Alternativas de Energía") de la Universidad Tecnológica Nacional Facultad Regional Bahía Blanca (UTN FRBB), una nueva metodología para la evaluación del proceso de enseñanza-aprendizaje, en reemplazo experimental de la vigente hasta ese momento en dicha materia, la cual consistía sólo en la realización de un proyecto final de cátedra para la evaluación de los conocimientos adquiridos.

La nueva metodología se diseñó considerando los criterios cognitivo constructivista y ecológicocontextual (Fernández y Granero, 2008; De León y Suárez, 2007), no utilizados comúnmente en carreras de ingeniería, ni en asignaturas de contenido técnico. Dicha herramienta utiliza no sólo los resultados de las evaluaciones de los alumnos, sino también una herramienta de evaluación de cátedra consistente en una planilla a ser completada por los estudiantes, una vez finalizada la etapa de evaluación del cursado de la asignatura.

El reemplazo de la metodología de evaluación vigente hasta el 2007 obedeció, entre otras cosas, al excesivo intervalo de tiempo observado entre el cursado de la asignatura y la entrega del proyecto, demorando su aprobación, con la consecuente repercusión, para el alumno, en el cursado de otras asignaturas de la carrera. Otro motivo tenido en cuenta fue la escasa cantidad de consultas realizadas por parte de los alumnos durante la ejecución del proyecto, evidenciando el poco acercamiento docente-alumno existente hasta ese momento, lo que fue considerado por la cátedra como síntoma de una inadecuada comunicación, que debía mejorarse.

La implementación se realizó simultáneamente en otra asignatura del Plan de la Carrera, "Generación, Transmisión y Distribución de la Energía Eléctrica", con la finalidad de ampliar la población de observación. Su contenido mayoritario consiste en 1) la descripción de las políticas de tarifas, precios y costos marginales de generación, transporte y distribución de la energía eléctrica, 2) la presentación de los principales tipos de centrales convencionales y no convencionales, ciclos e instalaciones propias de las centrales y las necesarias para la interconexión con las líneas, 3) el análisis de las leyes vigentes y normas de aplicación para la confección de proyectos a nivel Provincial y Nacional, tanto de Generación como en Transmisión y Distribución, 4) el cálculo diseño y proyecto de los componentes utilizados en los sistemas de transmisión y distribución de la energía eléctrica, como ser: líneas eléctricas de transmisión y de distribución, estaciones transformadoras y centros de distribución, urbanos y rurales, puestas a tierra, protecciones de líneas, redes y estaciones transformadoras.

Dicha asignatura fue elegida por compartir el mismo método de aprobación (basada en un proyecto final) que la asignatura "Fuentes No Convencionales de la Energía". El fundamento teórico de la metodología implementada está basado en el trabajo doctoral de Blanco Gutiérrez (2003), cuyos conceptos se transcriben oportunamente a lo largo del presente trabajo. 


\section{ASIGNATURA DE APLICACIÓN}

La asignatura Fuentes No Convencionales de la Energía (hoy denominada "Fuentes Alternativas de Energía") es una asignatura electiva del quinto año de la Carrera Ingeniería Electricista de la UTN FRBB. Su contenido mayoritario consiste en la descripción y el cálculo de sistemas eólicos e implementaciones fotovoltaicas, con una introducción descriptiva de otros sistemas que utilizan recursos renovables para generación de energía eléctrica (Castro et al., 1994; Colmenar et al., 2004).

Desde el año 2001, la modalidad de enseñanza fue la exposición oral y escrita de los temas por parte del docente a cargo. La evaluación de los alumnos para el cursado regular no contemplaba exámenes parciales, y la aprobación de dicha asignatura estaba sujeta a la ejecución de un proyecto final, cuyo tema era fijado por la cátedra.

De lo anterior puede observarse claramente que el criterio de enseñanza utilizado, cuadraba en cierta forma dentro del paradigma o criterio transmisión-recepción (o conductista), en el cual el profesor es considerado como una persona dotada de competencias aprendidas que transmite conforme a una planificación que se realiza en función de objetivos específicos, y el alumno es considerado como un receptor de las informaciones, cuya su misión es aprenderse lo que se le enseña (Blanco Gutiérrez, 2003), y el sentido de la evaluación se centra en el producto. Según Domínguez y Diez (1996), bajo este paradigma "la evaluación tiene como propósito recoger los resultados finales del proceso y valorar la eficacia del mismo en función de los porcentajes de obtención de los objetivos prefijados". En el marco del enfoque transmisión-recepción, se parte del supuesto de que todos los alumnos son iguales y, por lo tanto reciben la misma información, se los evalúa de la misma manera, con los mismos instrumentos y pautas establecidas para calificarlos.

La intención original no fue seguir este criterio, ya que la elección del proyecto de cátedra como forma de evaluación, pretendía asegurar el diálogo docente-alumno a través de la consulta en clase y fuera de ella, e ir evaluando el proceso de enseñanza-aprendizaje. Sin embargo, las consultas por parte de los alumnos fueron casi inexistentes durante el cursado, y el tiempo que transcurría entre la finalización del dictado de la asignatura y la entrega del proyecto era excesivo, demorando la aprobación de la materia, y retrasando al alumno en otras asignaturas de la carrera.

\section{MODIFICACIÓN DEL MÉTODO EN BASE A LOS MODELOS EDUCATIVOS}

Con la finalidad de revertir la situación anterior, se decidió modificar tanto el método de enseñanza como el de evaluación. Para el diseño del método, se tuvieron en cuenta conceptos de los paradigmas cognitivo constructivista y ecológico-contextual. Éstos, junto el paradigma conductual ya mencionado, han sido utilizados en el siglo XX para orientar los aspectos psicopedagógicos de modelos educativos en lo que respecta a cómo se concibe la enseñanza, el aprendizaje, y la manera de evaluar.

\section{El modelo cognitivo constructivista}

Blanco Gutiérrez (2003), señala que el modelo cognitivo de enseñanza se centra en los procesos de aprendizaje del sujeto que aprende, por lo tanto, se debe partir de las habilidades y estrategias básicas que el alumno domina, y de los modelos conceptuales que posee. El profesor cumple un papel de mediador, y el alumno posee un potencial de aprendizaje que puede desarrollar por medio de la interacción profesor-alumno (Fernández y Granero, 2008). De este modo, el modelo didáctico debe estimular la participación activa del alumno en su propio aprendizaje. Para este enfoque, la evaluación del alumno debe ser de tipo formativo y clínico; es decir, debe servirle al alumno para detectar errores de aprendizaje (de modo de corregir conceptos y posturas erradas), tomándolos como base para la adquisición de nuevos conocimientos (Stenhouse, 1984). La evaluación del proceso de enseñanza-aprendizaje (evaluación de cátedra) debe ser vista como una parte integrante del mismo, y no como el acto terminal. Rosales (1990), indica al respecto "que la evaluación forma parte indisociable de la enseñanza"..."y actúa como un instrumento de 
autorregulación y perfeccionamiento dentro del proceso instructivo". Román y Diez (1992) añaden que la evaluación "valora tanto los procesos como los productos, y será preferentemente formativa y criterial".

\section{El modelo ecológico-contextual}

Blanco Gutiérrez (2003), expresa que, sin menospreciar los aportes de la psicología cognitivista y el constructivismo como marco explicativo del aprendizaje, ha ido ganando terreno el enfoque educativo que estudia las relaciones que se establecen entre el sujeto y el entorno en el cual se desarrollan las actividades.

Dentro del enfoque contextual, el ambiente de clase ha de potenciar el conocimiento de todas las personas del grupo humano y el acercamiento de unos hacia otros (De León y Suárez, 2007). Progresivamente ha de posibilitar la construcción de un grupo humano cohesionado con objetivos y metas comunes (Bruner, 1997).

En su tesis doctoral, Blanco Gutiérrez (2003) señala que el proceso de evaluación de los alumnos debe ser realizado como algo natural que forma parte de las actividades ordinarias de clase, y no como una actividad especial que se realiza en un momento determinado del proceso de enseñanza-aprendizaje. La participación del alumno en su propia evaluación y la de sus compañeros es de importancia en este enfoque.

\section{DISEÑO DEL NUEVO MÉTODO}

El nuevo método implementado de evaluación del proceso de enseñanza-aprendizaje considera tanto los resultados de las evaluaciones de los alumnos, como la información recogida a través de la evaluación de cátedra.

\section{Evaluación del Alumno}

La evaluación del alumno se realiza, durante el cursado de la asignatura, mediante:

1. Exposiciones orales individuales para evaluar predominantemente contenidos factuales y actitudinales, y competencias psicolingüísticas. En la calificación del alumno orador intervienen indirectamente los alumnos que actúan como oyentes (Domínguez y Diez, 1996). Para ello, se toma en cuenta el nivel de interés logrado en la audiencia y el tipo de consultas derivadas de la exposición. Los oyentes son evaluados, a su vez, por el contenido de sus consultas y la actitud asumida durante la clase. Normalmente se realiza una sola exposición por alumno. Específicamente, al final de la clase se completa un registro en el cual figura el grado de participación de la clase (Ej. 20,50,70\%, etc.) y el interés expresado en responder las preguntas (MALO, REGULAR, BUENO, MUY BUENO). De esta forma se tiene una estadística que al final de cuatrimestre, permite a la cátedra cuantificar el interés de cada tema dictado, y además correlacionar el porcentaje promedio de participación con el porcentaje de cursado, evaluando así la efectividad de dictado

2. Pruebas escritas individuales, a través de:

a) Cuestionarios temáticos, sobre la base de los contenidos factuales, conceptuales, y procedimentales.

b) Análisis de casos, resolución de problemas, simulaciones y monografías realizados por el alumno en casa, con horarios para la realización de consultas, donde se evalúan principalmente contenidos conceptuales, procedimentales y actitudinales.

3. Informes escritos grupales, para el caso de: visitas, seminarios y proyectos, entregados al momento de rendir el examen final de promoción de la materia. 
Cada evaluación (oral o escrita) se realiza al finalizar un tema y se corrige con una puntuación del 0 al 100, y se aprueba con 70. Aquélla en la que el alumno obtenga menos de 70 debe recuperarla durante el examen final, caso contrario no aprueba el final.

En cada uno de los tres tipos de evaluación durante el cursado (exposición oral, cuestionario o problemas), se saca un promedio para ver en cuál de ellos el alumno presenta la nota más baja (es decir, las mayores dificultades). Aquel tipo de evaluación con el promedio más bajo es el que se incluye en el examen final si dicho promedio es menor a 70, junto con la presentación de un informe escrito grupal y la recuperación de la evaluación oral o escrita desaprobada durante el cursado.

Las evaluaciones escritas no tienen frecuencia prefijada de realización, pero sí fecha de presentación, penalizándose las entregas tardías con baja de puntaje o reprobación, según el caso. Por cada día de atraso en la entrega se baja el puntaje final en 5 puntos (es decir 5 puntos por cada día de atraso)

El examen final, oral y/o escrito, evalúa los contenidos conceptuales y procedimentales no alcanzados por las evaluaciones realizadas durante el cursado. La calificación obtenida se promedia con la del informe y las evaluaciones del cursado, siendo representativa del desempeño integral del alumno a lo largo del proceso completo de enseñanza-aprendizaje.

\section{Evaluación de cátedra.}

\section{1) Indicadores de medición}

Para cuantificar la calidad del proceso de enseñanza/aprendizaje a través de las evaluaciones del alumno, se definieron los indicadores que se enumeran a continuación. Dichos indicadores fueron elegidos porque demostraron ser los más efectivos a la hora de cuantificar la efectividad de la enseñanza de la materia, después de evaluar y probar múltiples alternativas (no descritas aquí):

1. De CUMPLIMIENTO: porcentaje de trabajos entregados en fecha (multiplicado por 100).

2. De APROBACIÓN DEL TEMA: porcentaje de evaluaciones aprobadas, por tema (multiplicado por 100).

3. De COMPRENSIÓN DEL TEMA: promedio del total de las calificaciones, por tema (de 0 a 100).

4. De CALIDAD ESCRITA: promedio de calificaciones del total de las evaluaciones escritas (de 0 a 100).

5. De NIVEL ESCRITO: porcentaje de evaluaciones escritas aprobadas (multiplicado por 100).

6. De CALIDAD EXPRESIÓN ORAL: promedio del total de las calificaciones de las evaluaciones orales (de 0 a 100).

7. De NIVEL EXPRESIÓN ORAL: porcentaje de exposiciones aprobadas (multiplicado por 100).

8. De CALIDAD ACADEMICA: calificaciones promedio de TODAS las evaluaciones (escritas y orales, de 0 a 100).

9. De NIVEL ACADÉMICO: porcentaje de evaluaciones TOTALES aprobadas (multiplicado por 100).

10. De MEJORA, por tipo: tendencia secuencial del indicador de COMPRENSIÓN DEL TEMA, sólo correspondiente a las calificaciones de evaluaciones escritas, segregadas por tipo 
(cuestionarios, análisis de casos, simulaciones, monografías, informes, resolución de problemas).

11. De MEJORA integral: tendencia secuencial del indicador de COMPRENSIÓN DEL TEMA.

Como puede observarse, los valores de todos los indicadores de medición están comprendidos entre 0 y 100 . La eficacia del proceso de enseñanza-aprendizaje se establece considerando el puntaje exhibido en la tabla 1.

Para que la calificación sea representativa, los valores de TODOS los indicadores deben caer en la misma categoría (1, 2, 3 ó 4). De no ser así, se adopta la categoría correspondiente al indicador de valor más bajo.

Tabla 1: Calificación del proceso enseñanza-aprendizaje.

\begin{tabular}{|c|c|c|c|}
\hline Categoría & Valor del indicador de medición & Puntuación & Calificación del método \\
\hline 1 & Más de 80 inclusive & Excelente & Eficaz \\
\hline 2 & Menos de 80 y hasta 70 inclusive & Buena & Aceptable \\
\hline 3 & Menos de 70 y hasta 40 inclusive & Regular & Ineficaz \\
\hline 4 & Menos de 40 & Mala & Ineficaz \\
\hline
\end{tabular}

\section{2) Planilla de evaluación de cátedra}

Si bien la modalidad de evaluación del alumno facilita el seguimiento cercano del proceso de enseñanza/aprendizaje, permitiendo corregir eventuales desajustes sobre la marcha, se incluye una planilla de evaluación de cátedra para recabar la opinión de los alumnos en relación a su experiencia en el cursado de la asignatura. La planilla es completada por el alumnado, una vez finalizada la etapa de evaluación del cursado de la asignatura (de modo que el alumno no se sienta presionado en sus respuestas por temor a ser excluido del cursado por sus opiniones), y aplicada para la mejora del proceso de enseñanza.

La planilla de evaluación de cátedra consiste en una guía de preguntas cuidadosamente confeccionada, que le permiten al alumno analizar:

1. Su grado de crecimiento académico, técnico y humano durante el cursado de la materia, en relación a la adquisición de nuevos conocimientos y aptitudes, y a su integración con el resto del grupo.

2. El desempeño de los docentes.

3. Su grupo de compañeros.

4. La calidad y profundidad que él percibe en cuanto al dictado del contenido del programa de la asignatura.

La guía de preguntas está diagramada para que el alumno evalúe, con una calificación entre Excelente, Muy bueno, Aceptable, Pobre y Deficiente: 
1. Si el cursado de la asignatura le sirvió al alumno para ser más capaz de: adquirir conocimientos y conceptos útiles; relacionar conceptos y cuestiones de la disciplina; resolver problemas; trabajar en equipo; plantear sus dudas; compartir y respetar otras opiniones; hacer trabajos / informes personales; comprender y aplicar consignas; organizar y auto-gestionar sus tiempos; leer y resumir; buscar y procesar información; elegir y manejar técnicas, programas o herramientas; ganar confianza en sí mismo/a; aprender a expresarse y a hacerse comprender; trabajar con responsabilidad y libertad.

2. El nivel de integración, participación, convivencia, diálogo alcanzado por el grupo de compañeros.

3. En los docentes: la transmisión cualitativa y cuantitativa de los contenidos; la planificación de las clases y las actividades; la forma y estrategia de presentar los temas; la claridad para explicar, dar consignas e instrucciones; la adecuación a la realidad del contenido de la materia; la destreza para preguntar y adecuar las preguntas; la destreza para descubrir las dificultades de comprensión del alumno; la habilidad para estimular la respuesta adecuada en los alumnos; la adecuación de sus respuestas a preguntas relevantes; la actitud frente a comentarios e inquietudes; la destreza en el uso de claves no verbales (p.ej. gestuales o de movimiento) para comunicar valor y significado; la capacidad para mantener el orden / la disciplina; la disponibilidad; el aspecto personal; la puntualidad; el diálogo (respeto / escucha a los alumnos); la capacidad para animar y motivar; la guía y el acompañamiento.

4. En relación a los contenidos: la cantidad, la calidad, la profundidad, la amplitud, la secuenciación, la integración teoría/práctica.

5. En cuanto a las actividades, los trabajos prácticos, el trabajo en aula y en campo, las visitas, los apuntes y el material de estudio: la adecuación de los tiempos de clase, la adecuación con los tiempos fuera de clase, y la relación entre lo enseñado y lo evaluado.

\section{RESULTADOS DE LA EXPERIENCIA}

La aplicación experimental del método en ambas asignaturas entregó valores por encima de los 80 puntos en los indicadores de medición. Desde un punto de vista cualitativo, la respuesta de los estudiantes fue excelente, lográndose una fluida comunicación docente-alumno, evidenciada en las consultas espontáneas al término de las clases, y la concurrida asistencia a los horarios de consulta establecidos.

Fue posible identificar errores en el dictado de los contenidos no detectados en años anteriores, y conocer el origen de las dificultades de los alumnos en relación a la comprensión de los contenidos, que anteriormente se evidenciaba sólo a través de las calificaciones. El método propició el acercamiento espontáneo de los alumnos, aún después de finalizado el cursado de la materia, quienes concurrían para facilitar material adicional de interés para la cátedra, y a la vez compartir sugerencias e inquietudes.

\section{CONCLUSIONES}

Los resultados obtenidos en la aplicación experimental demostraron la eficacia de la nueva metodología basada en los paradigmas cognitivo constructivista y ecológico-contextual. A fin de obtener mayor cantidad de datos para asegurar la confiabilidad de los resultados, se prevé continuar con la nueva modalidad, realizando un seguimiento con diferentes grupos de alumnos.

Es oportuno aclarar que la cantidad de alumnos durante la experiencia no sobrepasó de 8 (ocho) estudiantes. Debido a que el método presenta la desventaja de demandar un considerable esfuerzo docente debido a la forma de evaluación, su implementación sólo es factible si el grupo no supera las 10 personas, o si se cuenta con un docente evaluador cada 10 alumnos. 


\section{REFERENCIAS}

Blanco Gutiérrez O.; Estrategias de evaluación que utilizan los docentes de la carrera de Educación básica integral de la universidad de los Andes-Tachira, Tesis Doctoral; Universitat Rovira i Virgili, Departamento de Pedagogía; Tarragona (2003).

Blanco Gutiérrez O.; Tendencias en la evaluación de los aprendizajes, Revista de Teoría y Didáctica de las Ciencias Sociales: ISSN 1316-9505. Enero-Diciembre. 9, 111-130, MéridaVenezuela (2004).

Bloom B., J. Thomas y G. Madaus; Evaluación del Aprendizaje, Ed. Troquel, Argentina (1982).

Bruner, J. S.; La educación, puerta de la cultura, Madrid (1997).

Castro, M. y otros cinco autores; Desarrollo de la Docencia de las Energías Renovables en la UNED. VII CIES, VII Congreso Ibérico de Energía Solar, Energías Limpias en Progreso, (Bianual), págs. 733-738 (6), Organizador: ISES (International Solar Energy Society) - AEDES (Asociación Española de Energía Solar), Editores: Vázquez, M. y Morán, J. Universidad de Vigo. ISBN: 84605-0048-9, Dep. legal: C-886-94, Vigo, Pontevedra (España) (1994).

Colmenar, A., J. Peire y M. Castro; Energías Renovables para el Nuevo Milenio. Enseñanza a través de Sistemas Multimedia, XII CIES. XII Congreso Ibérico y VII Congreso Iberoamericano de Energía Solar, Siguiendo el Camino de las Estrellas, (Bianual), págs. 1309-1314 (6). Organizador: ISES (International Solar Energy Society) - AEDES (Asociación Española de Energía Solar), ISBN: 84-609-2260-X, Dep. legal: VG-779-2004, Vigo, Pontevedra (España) (2004).

De León I.C. y J.N. Suárez; Diseño instruccional y tecnologías de la información y la comunicación. Algunas reflexiones, Revista de Investigación: 1(61), ISSN 1010-2914, Venezuela (2007).

Domínguez G. y G. Diez; La Evaluación del Funcionamiento de un Centro a través del análisis de su Cultura Organizativa como Instrumento para la Mejora y la Innovación, Manual de Organización de Instituciones Educativas (347-404), Escuela Española, Madrid (1996).

Fernández Sola C. y J. Granero Molina: Planificación y guía docente en el marco de la convergencia europea de educación superior, Invest Educ Enferm.: 26 (2 supl): 128-135 (2008).

Gonzáles, O.; Cambiar el Sistema de Evaluación, significa un cambio de mentalidad, Materiales para el curso Evaluación Constructivista, Venezuela (1996).

Román R. y E. Diez; Currículum Vitae y Aprendizaje. Un modelo de diseño curricular de aula en el marco de la reforma, Ed. Itaka, Madrid (1992).

Rosales C.; Evaluar es Reflexionar sobre la Enseñanza, Ed. Narcea, Madrid (1990).

Stenhouse L.; Investigación y desarrollo del currículum. Madrid (1984). 